\title{
Effects of alcohol on food and energy intake in human subjects: evidence for passive and active over-consumption of energy
}

\author{
Martin R. Yeomans* \\ Department of Psychology, School of Life Sciences, University of Sussex, Brighton BN1 9QG, UK
}

\begin{abstract}
The effects of alcohol on food and energy intake in human subjects have been the subject of a number of controlled studies recently. Unlike the evidence for other macronutrients, there is minimal evidence for any compensatory reduction in food intake in response to energy ingested as alcohol. In contrast, all studies testing intake within $1 \mathrm{~h}$ of preload ingestion report a higher intake of food following alcohol relative to energy-matched controls, although this short-term stimulatory effect is not evident if the test meal is delayed beyond $1 \mathrm{~h}$. This time-course suggests that short-term stimulation of appetite may be mediated by the pharmacological action of alcohol on the appetite control system, either through enhanced orosensory reward or impaired satiety. In the long term, energy ingested as alcohol is additive to energy from other sources, suggesting that moderate alcohol consumption results in long-term passive over-consumption alongside shortterm active over-consumption of energy through appetite stimulation. Despite the consistency of enhanced energy intake after moderate alcohol, evidence of an association between alcohol in the diet and obesity remains contentious, although the most recent results suggest that alcohol intake correlates with BMI. Future research needs to address this issue and clarify the mechanisms underlying appetite stimulation by alcohol.
\end{abstract}

Alcohol: Energy intake: Food intake

Alcohol is a unique component of the human diet. First, alcohol can be considered to be a macronutrient providing $29 \mathrm{~kJ} / \mathrm{g}$, second only to fat in energy density. Second, alcohol acts pharmacologically on the nervous system, primarily by activating $\gamma$-aminobutyric acid receptors, but with effects on many other neurochemical systems. Both types of action are relevant to effects on food intake: the first since energy consumed as alcohol may modulate other sources of energy intake, and the second potentially having both direct and indirect effects on controls of food intake. The present review contrasts these two consequences of alcohol consumption and revisits the longstanding question of whether alcohol is a risk factor for obesity. The present review concentrates on acute and long-term effects of alcohol on energy intake, but a similarly detailed evaluation of alcohol on energy expenditure and metabolism is beyond the scope of the present review, and has been subject to detailed review (Lieber, 2000).

\section{Alcohol and short-term food intake}

Most studies of the short-term effects of alcohol on appetite in the laboratory have examined fixed amounts of an alcoholic drink consumed with a test meal or as a preload before a test meal. Contrasts of appetite measures between alcohol and control conditions then elucidate the effects of alcohol. Both the flavouring and post-ingestive effects of alcohol make adequate disguise of the alcohol preload problematic, and the most controlled studies have contrasted effects of the same product with and without alcohol in an alcohol-related context (Mattes, 1996; Poppitt et al. 1996; Hetherington et al. 2001) or in a non-alcoholic beverage (e.g. Yeomans et al. 1999). Less well-controlled studies have failed to disguise the alcohol administration (e.g. Tremblay \& St-Pierre, 1996). In all studies to date, alcohol taken before or with a meal has consistently failed to reduce short-term food intake (e.g. Foltin et al. 1993; Mattes, 1996; Poppitt et al. 1996). Indeed, alcohol results in a greater overall intake of energy from food during a test meal than in equivalent no-alcohol conditions (Tremblay et al. 1995; Tremblay \& St-Pierre, 1996; Westerterp-Plantenga \& Verwegen, 1999; Yeomans et al. 1999; Hetherington et al. 2001; Buemann et al. 2002; Yeomans \& Phillips, 2002).

The degree of compensatory eating following a macronutrient preload depends on a variety of factors, including the macronutrient tested, preload volume and energy density, time between preload and test meal, and sensitivity of the test meal. Timing may be particularly crucial with alcohol, since pharmacological and nutrient effects follow different time-courses, with the pharmacological action occurring sooner and decreasing as alcohol is metabolised, whereas signals arising from metabolism of alcohol consequently occur later. Thus, in acute studies, a short-delay between alcohol administration and appetite test may measure more the pharmacological effects, and longerterm studies more the metabolic consequences. Since all 
the studies reporting enhanced food intake after alcohol used short (typically 15-30 min) delays between alcohol consumption and appetite test (Tremblay et al. 1995; Tremblay \& St-Pierre, 1996; Westerterp-Plantenga \& Verwegen, 1999; Yeomans et al. 1999; Hetherington et al. 2001; Yeomans \& Phillips, 2002), or with alcohol consumed alongside the test meal (Buemann et al. 2002), the implication is that this reflects primarily a pharmacological action of alcohol. In contrast, longer-term studies have reported increased energy intake both for alcohol and energy-matched controls relative to a no-energy drink (Foltin et al. 1993; Mattes, 1996), implying that any short-term pharmacological enhancement of food intake by alcohol is compensated for by subsequent reductions in eating. However, some shorter-term studies reported no differences in short-term food intake following alcohol and energy-matched carbohydrate drinks (Poppitt et al. 1996, 1998). Since these studies also failed to find effects with carbohydrate preloads, whereas other studies have reported compensation for carbohydrate (French, 2004), this perhaps suggests a lack of sensitivity in the appetite test used in these studies. Longer-term diary-based studies show consistently that moderate alcohol intake is associated with higher daily energy intake relative to alcoholfree days, with energy derived from alcohol supplementing, rather than replacing, energy from food (de Castro \& Orozco, 1990), implying that the short-term stimulation of food intake by moderate alcohol consumption may be compensated for at later meals. In contrast to results from moderate alcohol intake, excessive alcohol intake is associated with lower intake of energy from fat and carbohydrate (e.g. Windham et al. 1983). Interpretation of results from individuals with high alcohol intake has to be viewed with caution, however, since alcoholism causes a wide range of liver and other physiological disturbances; thus, reduced food energy intake in alcoholism may be a consequence of the disease process rather than an accurate measure of effects of alcohol on appetite. Overall, although moderate alcohol may stimulate food intake in the short term, the overall effects of alcohol on energy balance appear to be more a consequence of failure to reduce other sources of intake in response to the energy in alcohol (passive over-consumption), coupled with effects on lipogenesis, rather than stimulation of additional intake of food energy.

\section{How might alcohol stimulate appetite?}

The mechanisms underlying the short-term stimulation of appetite by alcohol remain unclear, and a number of alternative hypotheses have been postulated. One possibility, that alcohol increases appetite per se, can be discounted, since rated appetite at the start of a test meal is unaffected by alcohol (e.g. Poppitt et al. 1998; Westerterp-Plantenga \& Verwegen, 1999; Hetherington et al. 2001) or has even been shown to be reduced after a preload containing alcohol (Yeomans et al. 1999). Thus, enhanced food intake following alcohol appears to result from some effect on feedback mechanisms resulting from food consumption, by enhancing hedonic (positive feedback) components of appetite control, or conversely, by impairing satiation (negative feedback). There is evidence to support both possibilities. In terms of positive feedback, it has been reported that hunger increases more during the early phase of a test meal following an alcohol preload than after an energy-matched carbohydrate preload (Yeomans et al. 1999; Fig. 1(A)). This pattern of response is similar to that seen when the palatability of food is modified through flavour manipulation (Yeomans, 1996). Moreover, increases in short-term food intake through flavour manipulation are compensated for later during the test day, at least in normal-weight unrestrained volunteers (Yeomans et al. 2001), a result analogous to the effects of alcohol discussed earlier. In addition, manipulated palatability enhances eating rate, as does alcohol (WesterterpPlantenga \& Verwegen, 1999). However, the implication that food may be more palatable following alcohol is not supported, since rated palatability has not been found to be greater after alcohol, although there has been a trend for higher palatability (Yeomans et al. 1999).

The alternative view, that alcohol decreases satiation, is supported by two findings. First, the rate of decrease of hunger during a meal was slower after alcohol than after an energy-matched control (Yeomans \& Phillips, 2002; Fig. 1(B)), and second, satiation had a later onset after alcohol (Westerterp-Plantenga \& Verwegen, 1999).
(A)

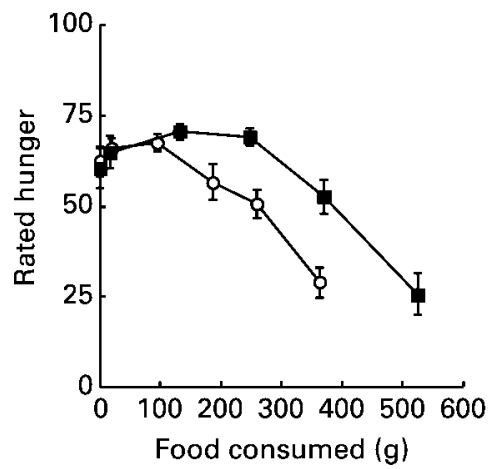

(B)

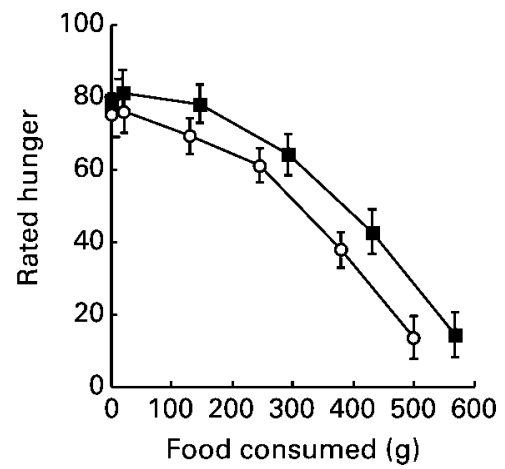

Fig. 1. Hunger ratings during a test meal following preloads of alcohol ( $\square)$ or an energy-matched control (O) when presented in a context unrelated to alcohol ((A), juice) and an alcohol context ((B), beer). (From Yeomans et al. (1999) and Yeomans \& Phillips (2002) respectively.) 
The contrast between the two studies shown in Fig. 1 warrants discussion. In one case, when participants were both unaware of the presence of alcohol and consumed alcohol in a neural context (Fig. 1(A)), the pattern of appetite was consistent with alcohol modifying palatability. In contrast, when actual alcohol remained undetected, but there was a cue (beer flavour) predicting alcohol (Fig. 1(B)), alcohol appeared to enhance satiation. The difference appears to lie in the context in which alcohol was delivered, implying that expectation of alcohol may mask its pharmacological action. A recent study contrasting energy intake when water, beer or wine were provided either ad libitum or as a fixed quantity with a meal (Buemann et al. 2002) further illustrates the complexity of these studies. Under ad libitum conditions, energy intake from all sources was only greater when wine was consumed with the meal, whereas there was a tendency for increased energy intake in the fixedvolume beer, but not wine, condition. The lack of a control drink with an alcohol cue, but no alcohol, limits conclusions about the nature of alcohol effects; until more studies control for context, clear conclusions cannot be drawn about the true nature of the effects of alcohol.

A third mechanism through which alcohol may enhance short-term food intake is through an indirect effect, such as disinhibition of restrained eating (Polivy \& Herman, $1976 a, b)$. Thus, in studies that did not control for restraint, intake after alcohol may have increased in participants who are restrained through removal of their cognitive self-restriction of eating. It is clear that this explanation is inadequate as a full explanation of the effects of alcohol, since in some studies only unrestrained participants responded to alcohol (Yeomans et al. 1999). The key factor here is not the post-ingestive effects of alcohol per $s e$, but the belief that alcohol has been consumed (Polivy \& Herman, 1976a). Disinhibited eating is only observed when participants believe they have consumed alcohol, and is interpreted in terms of alcohol interfering with the cognitive processes necessary to sustain dietary restraint (Polivy \& Herman, 1976b).

\section{Is moderate alcohol intake a risk factor for obesity?}

The evidence so far suggests that alcohol leads to overconsumption of energy, largely in a passive way. However, energy balance is a product of energy intake and expenditure, and for alcohol to be a risk factor for obesity the enhanced energy intake associated with moderate alcohol must exceed any consequent increase in metabolism. Despite several excellent reviews (Prentice, 1995; Suter et al. 1997), this issue remains contentious, with some maintaining that inefficient metabolism of alcohol means that enhanced energy intake is not associated with increased body-size, and others suggesting that energy from alcohol is a risk factor for obesity. The most recent studies tend to support the latter view, with BMI and alcohol intake positively correlated (Lahti-Koski et al. 2002; Wannamethee \& Shaper, 2003). In moderate consumers, it is possible that differences in energy metabolism or lifestyle differences that promote energy expenditure may partially disguise the consequences of passive overconsumption of energy derived from alcohol, and so confound earlier epidemiological studies. Alternatively, physiological effects of alcohol beyond its immediate effects on appetite may contribute to overall energy balance. For example, the case that alcohol is a risk factor for obesity in women remains weak (Prentice, 1995). Alcohol has an insulin-sensitising effect on skeletal muscle that down-regulates insulin secretion and alters lipogenesis. This in turn may reduce fat storage and has been suggested as an explanation for why women moderate drinkers tend to be leaner than non-drinkers (McCarty, 2000).

One of the issues raised during the live discussion after the presentation was the effect of confounders, e.g. evidence is suggested for more active people being moderate alcohol consumers, thus being able to offset the effect of increased energy intake due to alcohol.

\section{Conclusions}

Overall, studies to date are consistent with alcohol having a short-term stimulatory effect on appetite. This enhanced food intake, but not energy from alcohol, may be compensated for by reductions in subsequent voluntary food intake, but the failure to compensate for energy from alcohol does result in greater (passive) energy intake on days when alcohol is consumed. The extent to which this excess alcohol energy contributes to obesity, however, remains contentious, and both this issue and the mechanism underlying the short-term stimulatory effect of alcohol on appetite require further substantiation.

\section{References}

Buemann B, Toubro S \& Astrup A (2002) The effect of wine or beer versus a carbonated soft drink, served at a meal, on ad libitum energy intake. Int J Obes 26, 1367-1372.

De Castro JM \& Orozco S (1990) Moderate alcohol intake and spontaneous eating patterns of humans: evidence of unregulated supplementation. Am J Clin Nutr 52, 246-253.

Foltin RW, Kelly TH \& Fischman MW (1993) Ethanol as an energy source in humans: comparison with dextrose-containing beverages. Appetite 20, 95-110.

French S (2004) Effects of dietary fat and carbohydrate on appetite vary depending upon site and structure. Br J Nutr 92, Suppl. 1, S23-S26.

Hetherington MM, Cameron F, Wallis DJ \& Pirie LM (2001) Stimulation of appetite by alcohol. Physiol Behav 74, 283-289.

Lahti-Koski M, Pietinen P, Heliovaara M \& Vartiainen E (2002) Associations of body mass index and obesity with physical activity, food choices, alcohol intake, and smoking in the 1982-1997 FINRISK Studies. Am J Clin Nutr 75, 809-817.

Lieber CS (2000) Alcohol: Its metabolism and interaction with nutrients. Ann Rev Nutr 20, 395-400.

McCarty MF (2000) The insulin-sensitising activity of moderate alcohol consumption may promote leanness in women. Med Hypotheses 54, 794-797.

Mattes RD (1996) Dietary compensation by humans for supplemental energy provided as ethanol or carbohydrate in fluids. Physiol Behav 59, 179-187.

Polivy J \& Herman CP (1976a) The effects of alcohol on eating behavior: disinhibition or sedation? Addict Behav 1, $121-125$. 
Polivy J \& Herman CP (1976b) Effects of alcohol on eating behavior: influence of mood and perceived intoxication. $J$ Abnorm Psychol 85, 601-606.

Poppitt SD, Eckhardt JW, McGonagle J, Murgatroyd PR \& Prentice AM (1996) Short-term effects of alcohol-consumption on appetite and energy-intake. Physiol Behav 60, 1063-1070.

Poppitt SD, McCormack D \& Buffenstein R (1998) Short term effects of macronutrient preloads on appetite and energy intake in lean women. Physiol Behav 64, 279-285.

Prentice AM (1995) Alcohol and obesity. Int J Obes 19, S44-S50.

Suter PM, Hasler E \& Vetter W (1997) Effects of alcohol on energy metabolism and body weight regulation: Is alcohol a risk factor for obesity? Nutr Rev 55, 157-171.

Tremblay A \& St-Pierre S (1996) The hyperphagic effect of a high-fat diet and alcohol intake persists after control for energy density. Am J Clin Nutr 63, 479-482.

Tremblay A, Wouters E, Wenker M, St-Pierre S, Bouchard C \& Despres J-P (1995) Alcohol and a high-fat diet: a combination favoring overfeeding. Am J Clin Nutr 62, 639-644.

Wannamethee SG \& Shaper AG (2003) Alcohol, body weight, and weight gain in middle-aged men. Am J Clin Nutr 77, 1312-1317.

Westerterp-Plantenga MS \& Verwegen CRT (1999) The appetizing effect of an aperitif in overweight and normal-weight humans. Am J Clin Nutr 69, 205-212.

Windham CT, Wyse BW \& Hansen RG (1983) Alcohol consumption and nutrient density of diets in the Nationwide Food Consumption Survey. J Am Diet Assoc 82, 365-372.

Yeomans MR (1996) Palatability and the microstructure of eating in humans: the appetiser effect. Appetite 27, 119-133.

Yeomans MR, Hails NJ \& Nesic JS (1999) Alcohol and the appetiser effect. Behav Pharmacol 10, 151-161.

Yeomans MR, Lee MD, Gray RW \& French SJ (2001) Effects of test-meal palatability on compensatory eating following disguised fat and carbohydrate preloads. Int $J$ Obes $\mathbf{2 5}$, $1215-1224$.

Yeomans MR \& Phillips MF (2002) Failure to reduce short-term appetite following alcohol is independent of beliefs about the presence of alcohol. Nutr Neurosci 5, 131-139. 